\title{
Conhecimento dos manipuladores de alimentos sobre boas práticas nos restaurantes públicos populares do Estado do Rio de Janeiro
}

\author{
Knowledge of food handlers concerning good practices in \\ popular public restaurants in the State of Rio de Janeiro, Brazil
}

\section{Autores | Authors}

$\bowtie$ Aline Gomes de MELLO

Fundação Oswaldo Cruz (Fiocruz) Instituto Nacional de Controle de

Qualidade em Saúde

Av. Calos Chagas Filho prédio CCS, bloco J, $2^{\circ}$ andar salda 26

Cidade Universitária CEP 21941-590

Rio de Janeiro/RJ - Brasil e-mail: alinemrj@terra.com.br

Mauricio de Pinho GAMA

Universidade Federal do Rio de Janeiro (UFRJ)

Divisão de Pesquisa Hospital Universitário Clementino Fraga Filho e-mail:mpgama@hucf.ufrj.br

Victor Augustus MARIN

Fundação Oswaldo Cruz (Fiocruz) Instituto Nacional de Controle de Qualidade em Saúde

Departamento de Microbiologia e-mail: victor.marin@incqs.fiocruz.br

\section{Luciléia Granhen Tavares} COLARES

Universidade Federal do Rio de Janeiro (UFRJ)

Instituto de Nutrição Josué de Castro Departamento de Nutrição e Dietética e-mail: lucolares@terra.com.br

Autor Correspondente / Corresponding Author

Recebido / Received: 08/07/2009 Aprovado / Approved: 16/03/2010

\section{Resumo}

Os manipuladores são a principal via de contaminação dos alimentos, o que pode levar ao surto de doenças transmitidas por alimentos. Neste contexto, o objetivo deste estudo foi avaliar o nível de conhecimento dos manipuladores dos Restaurantes Públicos Populares (RPP) do Estado do Rio de Janeiro sobre boas práticas de manipulação de alimentos, relacioná-las à sua capacitação e esta com as condições higiênico-sanitárias dos RPP. Trata-se de estudo exploratório, realizado entre outubro/2007 e janeiro/2008 em dez RPP. Um questionário composto de 17 questões fechadas e abertas, sendo 8 relativas ao conhecimento sobre boas práticas de manipulação, foi aplicado a 103 manipuladores de alimentos. Foi aplicado o teste de correlação entre as varáveis nível de conhecimento dos manipuladores, capacitação nos RPP e condições higiênico-sanitárias dos RPP. O conhecimento dos manipuladores, em média, foi considerado regular (56,09\%), sendo que os RPP 3 e 10 obtiveram nível de conhecimento deficiente, respectivamente, 48,3 e 49,1\% de respostas corretas e em nenhum dos RPP foi constatado bom nível de conhecimento sobre boas práticas de manipulação. Houve correlação positiva entre a existência de capacitação e a avaliação higiênico-sanitária dos RPP, assim como com o nível de conhecimento. Conclui-se que foi regular o percentual de respostas corretas obtido sobre boas práticas, o que reforça que cabe investir na capacitação dos manipuladores de alimentos, sendo um desafio encontrar a melhor didática para esta prática de forma que não só atenda aos anseios dos trabalhadores, mas também às exigências da legislação sanitária.

Palavras-chave: Manipuladores de alimentos; Capacitação; Boas práticas de manipulação; Restaurantes.

\section{Summary}

The food handlers are the main contamination route for foods and can lead to food poisoning outbreaks. Thus the objective of the present study was to evaluate the level of knowledge of the food handlers working in popular public restaurants (PPR) in Rio de Janeiro/Brazil on good practices in food handling, and relate this to their training, and their training to the hygienic-sanitary conditions of the PPR. This was an exploratory study, carried out between October/2007 and January/2008 in ten PPR. A questionnaire consisting of 17 closed and open questions, of which 8 concerned their knowledge about good handling practices, was applied to 103 food handlers. Statistical tests were applied to test for correlation between the various levels of knowledge of the handlers, their training in the PPR and the hygienic-sanitary conditions of the PPR. On average, the knowledge of the food handlers was considered to be regular (56.09\%), although those working in PPR 3 and 10 showed poor levels of knowledge of, respectively, 48.3 and $49.1 \%$ of correct answers. None of the PPR showed good levels of knowledge concerning handling practices. There was positive correlation between the existence of training and the evaluation of the sanitary-hygienic conditions of the PPR, as well as the level of knowledge. It was concluded that the percentage of correct responses on good practices was regular, which reinforces the need to invest in the training of food handlers. Finding the best teaching methods for such training that not only meet the needs of the employees but also the requirements of the sanitary legislation, represents a considerable challenge. 


\section{Introdução}

A ingestão de alimentos contaminados por microrganismos patogênicos, substâncias químicas, objetos lesivos ou que contenham em sua constituição substâncias tóxicas que chegam ao nosso organismo acarretam o surgimento das Doenças Transmitidas por Alimentos (DTA) (OMS, 2002).

As DTA são um problema de Saúde Pública, que ocasionam a redução da produtividade, perdas econômicas e afetam a confiança do consumidor. Além disso, dependendo da quantidade do alimento contaminado ingerido, do tipo de microrganismo ou toxina e do estado de saúde do indivíduo acometido, as DTA, podem levar à mortalidade (BENEVIDES e LOVATTI, 2004).

Segundo Arruda (2000), a questão da segurança alimentar sob o ponto de vista higiênico-sanitário é de grande preocupação e por isso faz-se necessária a adoção de medidas que previnam a contaminação nas diferentes etapas do processo produtivo, uma vez que as DTA se propagam com rapidez e alta patogenicidade.

A incidência global de DTA é difícil de ser estimada, porém há relatos de que no ano de 2000 cerca de 2,1 milhões de pessoas no mundo foram a óbito devido a um quadro diarreico, sendo grande parte desses óbitos atribuída ao consumo de água e alimentos contaminados. Nos países industrializados, o percentual de pessoas que desenvolvem algum sintoma de DTA tem aumentado a cada ano. Nos EUA cerca de 76 milhões de casos de DTA resultam em 325.000 hospitalizações e 5.000 óbitos, anualmente (OMS, 2002).

Registros epidemiológicos mostram que os serviços de alimentação contribuem para o alto índice de doença transmitida por alimentos. Acredita-se que esses estabelecimentos sejam responsáveis por mais de $50 \%$ de ocorrência de surtos, contribuindo para este quadro os ambientes, os equipamentos, os utensílios e os manipuladores de alimentos (OMS, 2002).

Wei e Chiou (2002) identificaram que o surto causado por Staphylococcus aureus, que atingiu 10 estudantes de uma escola tailandesa, após a realização do desjejum, foi proveniente de um manipulador de alimentos que apresentava lesão em uma das mãos. Esse resultado vem reforçar a importância desses profissionais na contaminação dos alimentos.

O termo manipulador de alimentos, de acordo com a Organização Mundial de Saúde (OMS, 1989) “...incluyen a todas las personas que puedem entrar em contacto con un producto comestible o parte del mismo en cualquier etapa de las que van desde su fuente, por ejemplo, la granja, hasta el consumidor". A RDC 216/2004 classifica como manipulador de alimentos qualquer pessoa do serviço de alimentação que entra em contato direto ou indireto com o alimento (BRASIL, 2004).

Segundo a OMS (1989), o manipulador é a principal via de contaminação dos alimentos produzidos em larga escala e desempenha papel importante na segurança dos alimentos, na preservação da higiene dos alimentos durante toda a cadeia produtiva, desde o recebimento, armazenamento, preparação até a distribuição. Uma manipulação incorreta e o descuido em relação às normas higiênicas favorecem a contaminação por microrganismos patogênicos

Em trabalho desenvolvido por Barbosa Neta et al. (2004) em um Restaurante da Universidade Federal do Rio Grande do Norte, observou-se a falta de cuidado dos manipuladores de alimentos com as normas de higiene, entre elas o uso de uniforme incompleto, a não manutenção da limpeza dos sapatos e botas, as unhas grandes, as barbas e bigodes não aparados, o que os torna, portanto, uma fonte de contaminação dos alimentos.

Para verificar o nível de contaminação nas mãos dos manipuladores, Almeida et al. (1995) desenvolveram um estudo na cozinha de um Restaurante Universitário em Campinas, SP, que fornecia cerca de 6500 refeições por dia. Como resultado, os autores verificaram que os manipuladores avaliados raramente lavavam as mãos quando entravam na cozinha e durante o preparo dos alimentos.

Um estudo semelhante foi realizado por Lagaggio et al. (2002) em que pesquisaram microrganismos patogênicos nas mãos dos manipuladores de alimentos do Restaurante da Universidade Federal de Santa Maria antes e após a orientação quanto ao procedimento correto de higienização das mãos. Antes da orientação, 100\% das amostras estavam contaminadas e, depois, constatou-se a redução para $22,22 \%$ de mãos contaminadas. Os autores apontam que a capacitação quanto à forma correta de higienização das mãos foi uma medida eficaz na redução da contaminação dos alimentos servidos aos estudantes.

Em âmbito geral é grande a necessidade de melhorar a qualidade dos produtos e serviços, assim como capacitar os manipuladores de alimentos para que adquiram hábitos higiênico-sanitários adequados e os apliquem no dia a dia. De acordo com a RDC 216/2004 (BRASIL, 2004), os responsáveis pelas atividades de manipulação dos alimentos devem ser comprovadamente submetidos a curso de capacitação, que deve abordar, no mínimo, assuntos como: contaminantes alimentares, doenças transmitidas por alimentos, manipulação higiênica dos alimentos e boas práticas de manipulação.

O processo de capacitação deve ser contínuo a fim de facilitar a implantação de procedimentos de boas 
práticas de manipulação, que auxiliam na manutenção da qualidade das refeições produzidas.

Com o intuito de minimizar a fome e promover a segurança alimentar e nutricional, o Governo do Estado do Rio de Janeiro implantou, entre outros programas de segurança alimentar, o Programa Estadual de Acesso à Alimentação (PEAA), que é coordenado pelo Conselho Estadual de Combate à Fome. Fazem parte desse programa os Restaurantes Públicos Populares (RPP) (SEASDH, 2007).

Os RPP do Estado do Rio de Janeiro são geridos pela Secretaria de Estado de Assistência Social e Direitos Humanos (SEASDH) e são Unidades de Alimentação e Nutrição (UAN) que apresentam como objetivo a distribuição de refeições prontas, nutricionalmente balanceadas e originadas de processos seguros, servidas em locais apropriados, confortáveis, de fácil acesso e ao custo de $\mathrm{R} \$ 1,00$ (um Real) para o usuário (MDS, 2005).

A pesquisa realizada por Mello (2009) que avaliou as condições higiênico-sanitárias de 10 RPP a partir da aplicação de um roteiro de inspeção sanitária, em que foram considerados os aspectos que estavam em conformidade com a legislação sanitária (RDC 216/2004), aponta inadequação das condições higiênicosanitárias desses estabelecimentos, visto que 10\% foram classificados como bom, 50\% como regular e 40\% como deficiente.

Tendo em vista que, atualmente, no Estado do Rio de Janeiro, existem treze Restaurantes Públicos Populares (RPP) que servem juntos 12.375 cafés da manhã e 30.875 almoços, por dia, de segunda à sexta-feira, é importante que estes estabelecimentos possuam manipuladores de alimentos qualificados a fim de assegurar a qualidade das refeições produzidas (SEASDH, 2008).

Desta forma, o objetivo deste estudo foi avaliar o nível de conhecimento dos manipuladores dos Restaurantes Públicos Populares do Estado do Rio de Janeiro sobre boas práticas de manipulação de alimentos, relacioná-las com sua capacitação e estas com as condições higiênico-sanitárias dos RPP.

\section{Material e métodos}

Trata-se de estudo exploratório, seccional, desenvolvido no período de outubro de 2007 a janeiro de 2008 em dez (10) Restaurantes Públicos Populares do Estado do Rio de Janeiro, sendo quatro localizados no município do RJ (Herbert de Souza / Central do Brasil, Getúlio Vargas/Bangu, Jornalista Jorge Curi/Maracanã, João Goulart/Bonsucesso); um em Duque de Caxias (Dom Hélder Câmara); um em Niterói (Jorge Amado); um em Nova Iguaçu (Madre Tereza de Calcutá); um em Itaboraí (Governador Mario Covas); um em Campos dos
Goytacazes (Romilton Bárbara); e um em Barra Mansa (Irmã Ruth). A pesquisa foi aprovada pelo Comitê de Ética em Pesquisa com seres humanos do Núcleo de Estudos em Saúde Coletiva da Universidade Federal do Rio de Janeiro (CEPNESC/UFRJ), parecer número 33/2007 e autorizada pela Secretária de Assistência Social e Direitos Humanos do Estado do Rio de Janeiro.

Os 103 manipuladores de alimentos que participaram desta etapa da pesquisa foram selecionados por acessibilidade (GIL, 1998). Foi aplicado questionário aos manipuladores de alimentos dos RPP, por entrevistador treinado, no próprio restaurante, durante o horário de funcionamento com o objetivo de caracterizá-los com relação às condições sócio-econômicas (sexo, idade, escolaridade e a renda mensal) e aos conhecimentos sobre boas práticas de manipulação de alimento (Quadro 1). Antes de responder ao questionário, todos os participantes assinaram o termo de consentimento livre e esclarecido, de acordo com a aprovação da pesquisa pelo Comitê de Ética em Pesquisas com Seres Humanos do Núcleo de Estudos em Saúde Coletiva da Universidade Federal do Rio de Janeiro (CEPNESC - UFRJ, parecer 33/2007).

O questionário elaborado foi adaptado de Praxedes (2003) e Guimarães (2006) e constou de 17 questões fechadas e abertas, sendo que, destas, 7 questões estavam relacionadas às condições sócio-econômicas, 8 aos conhecimentos de contaminação dos alimentos, doenças transmitidas por alimentos e boas práticas de manipulação e 2 à capacitação do manipulador de alimentos.

Para comparar os resultados entre os homens e as mulheres em relação às condições sócio-econômicas, foi aplicado o teste $t$-student, considerando o nível de significância 5\%.

Para avaliar o nível de conhecimento sobre boas práticas de manipulação, as respostas foram categorizadas em: corretas; parcialmente corretas, quando havia algum termo correto; e incorretas, quando os manipuladores não sabiam responder ou quando respondiam incorretamente aos questionamentos, tomando por base Castro (2007). Os dados foram apresentados descritivamente através da média e desvio padrão.

Foi considerado correto um total de 824 respostas obtidas, levando em consideração o número de funcionários, de cada RPP, que responderam ao questionário multiplicado pelas 8 questões correspondentes.

Para classificar o nível de conhecimento sobre contaminação dos alimentos, doenças transmitidas por alimentos e boas práticas de manipulação, foi utilizado o parâmetro adaptado de Saccol (2007) e classificado como deficiente quando de 0 a $50 \%$ das respostas estavam corretas; regular quando de 51 a $75 \%$ das respostas 
Conhecimento dos manipuladores de alimentos sobre boas práticas nos restaurantes públicos populares do Estado do Rio de Janeiro

MELLO, A. G. et al.

Quadro 1. Questionário semiestruturado aplicado aos manipuladores de alimentos dos Restaurantes Públicos Populares.

1) Sexo: $\square$ Feminino $\square$ Masculino

\section{Questionário aplicado aos manipuladores de alimentos}

Características sócio-econômicas

2) Idade: $\square$ até 20 anos $\square$ 20-29 anos $\square$ 30-39 anos $\square$ 40-49 anos $\square$ acima de 50 anos

3) Escolaridade:

$\square$ ensino fundamental incompleto $\square$ ensino fundamental completo

口 ensino médio incompleto $\quad \square$ ensino médio completo

$\square$ ensino superior incompleto $\quad \square$ ensino superior completo

4) Naturalidade? $\square$ Rio de Janeiro $\square$ outro estado:

5) Restaurante onde está trabalhando: _________ Função:

6) Renda individual mensal (salário mínimo de $R \$ 380,00$ ) como referência ano de 2007.
๑ 1 salário mínimo
$\square 2$ salários mínimos
$\square 3$ salários mínimos
$\square 4$ ou mais salários mínimos

7) É seu primeiro emprego em restaurante? $\square$ sim $\square$ não

Caso não, Que função desempenhava anteriormente?

\section{Conhecimento sobre contaminação dos alimentos, doenças transmitidas por alimentos e boas práticas de manipulação}

1) Você já ouviu falar em contaminação dos alimentos? $\square$ sim $\square$ não

Em caso positivo, como acontece?

2) Em quais setores do restaurante pode ocorrer contaminação dos alimentos?

3) Com relação às mãos, elas podem contaminar o alimento? $\square$ sim $\square$ não

Em caso positivo, de que forma?

4) Você acha que os alimentos podem causar doenças? $\square \operatorname{sim}$ 口 não

5) Quais são as doenças causadas por alimentos?

6) Você acha importante ter uma boa higiene pessoal para trabalhar com alimentos?

口sim $\square$ não Por quê?

7) $\bigcirc$ que você considera importante na higiene pessoal?

8) O que são as Boas Práticas de Manipulação?

\section{Capacitação do manipulador de alimentos}

1) Você já participou de algum treinamento no trabalho? $\square$ sim $\square$ não

2) Quando foi o último treinamento de que você participou?

estavam corretas; e bom quando de 76 a $100 \%$ das respostas estavam corretas.

Para avaliar a relação entre o nível de conhecimento dos manipuladores de alimentos e a existência de capacitação, bem como a capacitação e o percentual de adequação das condições higiênico-sanitárias, dados apontados por Mello (2009) (Quadro 2), foi utilizada a Correlação Linear de Pearson, obedecendo à seguinte classificação (Quadro 3).

\section{Resultados e discussão}

Foram entrevistados 103 manipuladores de alimentos, que corresponde a $22,5 \%$ do total de funcionários que trabalham nos dez RPP, sendo, destes, $33 \%$ do gênero feminino e $67 \%$ do gênero masculino.

Com relação à idade, 64\% dos manipuladores de alimentos encontravam-se inseridos na faixa entre 30 e 49 anos, estando 33,3\% dos homens na faixa entre 30 e 39 anos e $38,2 \%$ das mulheres entre 40 e 49 anos.
Quanto ao nível de escolaridade, predominou o ensino fundamental incompleto (35\%). Outras pesquisas também apontam para um baixo nível de escolaridade dos manipuladores de alimentos (SILVA et al., 2003; PRAXEDES, 2003 e SOUZA et al., 2004). Silva et al. (2003), em pesquisa com indivíduos responsáveis pela merenda escolar de 24 unidades de ensino fundamental localizadas em São Paulo/SP, revelaram que, dos manipuladores de alimentos, apenas 12,5\% haviam concluído o ensino médio. Praxedes (2003) também observou que apenas $18,5 \%$ dos comerciantes ambulantes de alimentos possuíam o ensino médio. Dados semelhantes foram encontrados por Souza et al. (2004), confirmando a baixa escolaridade entre os manipuladores de alimentos.

$\mathrm{Na}$ presente pesquisa também se observou que $60,2 \%$ dos funcionários entrevistados recebiam um salário mínimo, sendo a média da renda dos homens significantemente maior que a das mulheres, 
MELLO, A. G. et al.

Quadro 2 . Percentual de adequação das condições higiênicosanitárias (PACHS) de dez restaurantes públicos populares do Estado do Rio de Janeiro.

\begin{tabular}{|cc|}
\hline RPP & PACHS (\%) \\
1 & 64,5 \\
2 & 33,7 \\
3 & 73,5 \\
4 & 66,9 \\
5 & 48,2 \\
6 & 37,3 \\
7 & 67,5 \\
8 & 78,9 \\
9 & 47,0 \\
10 & 71,1 \\
\hline
\end{tabular}

Fonte: Mello, 2009.

Quadro 3. Classificação da correlação linear de Pearson.

\begin{tabular}{cc|}
\hline Coeficiente de correlação & Correlação \\
$r=1$ & Perfeita positiva \\
$0,8 \leq r<1$ & Forte positiva \\
$0,5 \leq r<0,8$ & Moderada positiva \\
$0,1 \leq r<0,5$ & Fraca positiva \\
$0<r<0,1$ & Íntima positiva \\
0 & Nula \\
$-0,1<r<0$ & Íntima negativa \\
$-0,5<r \leq-0,1$ & Fraca negativa \\
$-0,8<r \leq-0,5$ & Moderada Negativa \\
$-1<r \leq-0,8$ & Forte negativa \\
$r=-1$ & Perfeita negativa \\
\hline
\end{tabular}

Fonte: Vieira, 1984.

embora possuíssem o mesmo nível de escolaridade e desempenhassem a mesma função ( $p$-valor < 0,05).

Resultado semelhante foi encontrado em estudo realizado por Castro (2007) em restaurantes comerciais do tipo self service, localizados em shopping centers do Município do Rio de Janeiro/RJ, que constatou que 89\% dos manipuladores recebiam até dois salários mínimos e que a maioria dos homens, mesmo apresentando baixo nível de escolaridade, recebia melhores salários que as mulheres.

Os baixos salários recebidos pelos manipuladores de alimentos dos diferentes segmentos do serviço de alimentação favorecem a grande rotatividade de mão de obra em busca de melhores salários, sendo este um dos fatores que dificulta a implantação das boas práticas de manipulação neste setor, conforme relatado por Silva Jr (2007).

Dos funcionários que trabalhavam nos RPP, $41,7 \%$ relataram que este era o seu primeiro emprego. Em pesquisa realizada por Guimarães (2006) com manipuladores de lojas de conveniência localizadas em postos de combustíveis da Cidade do Rio de Janeiro e que serviam refeições, para 66,7\% dos funcionários, este era o seu primeiro emprego e, dos que já haviam trabalhado anteriormente, apenas 10,3\% tiveram funções relacionadas à manipulação de alimentos. Em pesquisa realizada por Praxedes (2003) com comerciantes de alimentos, observou-se que somente $50 \%$ dos entrevistados haviam tido experiência anterior na área de alimentos.

Diante disso, a capacitação contínua dos manipuladores de alimentos é muito importante, tendo em vista que no setor de alimentação são contratados profissionais sem experiência na função que irá desempenhar.

Para Praxedes (2003), a comercialização de alimentos é uma alternativa viável em situação de desemprego, mas se caracteriza em risco devido ao baixo conhecimento sobre a manipulação higiênica dos alimentos, ignorando que estes podem servir de via de transmissão de doenças, o que reforça a necessidade de uma fiscalização sanitária bastante ativa e de caráter mais orientador do que punitivo.

Em relação à avaliação do nível de conhecimento dos manipuladores de alimentos dos RPP quanto à contaminação dos alimentos, doenças transmitidas por alimentos e boas práticas de manipulação, houve em média 56,09\% \pm 6,32 de respostas corretas (Tabela 1).

As respostas incorretas e parcialmente corretas estavam, principalmente, relacionadas com os seguintes temas: contaminação cruzada, higiene das mãos, doenças transmitidas por alimentos e a definição de boas práticas de manipulação.

Castro (2007), ao avaliar a percepção dos manipuladores quanto ao alimento seguro, constatou que apenas 4 dos 109 entrevistados tiveram percepção satisfatória e, destes, 50\% haviam sido capacitados anteriormente. A pesquisa enfatiza a importância da forma de abordagem dos conteúdos durante a capacitação para que o conhecimento seja adquirido e aplicado no dia a dia.

De acordo com Fernández et al. (1998), a prática educativa deve ser realizada em local agradável, de forma que não haja interrupções, o que facilita a integração do conhecimento e sua aplicação no cotidiano e ainda deve-se considerar os desejos e as necessidades dos participantes, assim como o nível de escolaridade e conhecimento sobre o tema a ser tratado.

$\mathrm{Na}$ presente pesquisa, a partir das respostas dos manipuladores, pode-se verificar que alguns deles têm a percepção de que o restaurante em que trabalha é muito limpo e por isso a contaminação dos alimentos não é passível de acontecer, visto que, o manipulador não consegue visualizar o processo produtivo como um todo, limitando sua avaliação apenas ao seu setor 
Tabela 1. Quantificação dos RPP em relação às respostas das 8 questões que abordam sobre higiene pessoal, contaminação dos alimentos, doenças transmitidas por alimentos e boas práticas de manipulação.

\begin{tabular}{|c|c|c|c|c|c|c|c|c|c|}
\hline \multirow[t]{2}{*}{ RPP } & \multicolumn{2}{|c|}{$\mathbf{N}^{\circ}$ de manipuladores } & \multicolumn{6}{|c|}{ Total de respostas obtidas } & \multirow{3}{*}{$\begin{array}{c}\text { Total de } \\
\text { respostas/RPP } \\
\mathbf{n}\end{array}$} \\
\hline & \multirow{2}{*}{$\begin{array}{c}\text { Que responderam } \\
\text { ao questionário } \\
\text { n }\end{array}$} & \multirow{2}{*}{$\begin{array}{c}\text { Capacitados que } \\
\text { responderam ao } \\
\text { questionário } \\
\text { n }\end{array}$} & \multicolumn{2}{|c|}{$\begin{array}{l}\text { Não sabe ou } \\
\text { incorretas }\end{array}$} & \multicolumn{2}{|c|}{$\begin{array}{c}\text { Parcialmente } \\
\text { corretas }\end{array}$} & \multicolumn{2}{|c|}{ Corretas } & \\
\hline $\mathbf{n}$ & & & $\mathbf{n}$ & $\%$ & $\mathbf{n}$ & $\%$ & $\mathbf{n}$ & $\%$ & \\
\hline 1 & 7 & 7 & 14 & 25 & 4 & 7,1 & 38 & 67,9 & 56 \\
\hline 2 & 8 & 3 & 25 & 39,1 & 6 & 9,4 & 33 & 51,6 & 64 \\
\hline 3 & 15 & 6 & 41 & 34,2 & 21 & 17,5 & 58 & 48,3 & 120 \\
\hline 4 & 6 & 6 & 10 & 20,8 & 8 & 16,7 & 30 & 62,5 & 48 \\
\hline 5 & 18 & 13 & 35 & 24,3 & 29 & 20,1 & 80 & 55,6 & 144 \\
\hline 6 & 9 & 1 & 19 & 26,4 & 14 & 19,4 & 39 & 54,2 & 72 \\
\hline 7 & 10 & 10 & 25 & 31,3 & 11 & 13,7 & 44 & 55 & 80 \\
\hline 8 & 10 & 9 & 22 & 27,5 & 8 & 10 & 50 & 62,5 & 80 \\
\hline 9 & 6 & 6 & 17 & 35,4 & 5 & 10,4 & 26 & 54,2 & 48 \\
\hline 10 & 14 & 11 & 38 & 33,9 & 19 & 18,3 & 55 & 49,1 & 112 \\
\hline Total & 103 & 72 & 242 & - & 124 & - & 449 & - & 824 \\
\hline$x$ & - & - & - & 29,79 & - & 14,14 & - & 56,09 & - \\
\hline $\mathrm{DP}$ & - & - & - & 5,84 & - & 4,76 & - & 6,32 & - \\
\hline
\end{tabular}

Legenda: $\mathrm{X}=$ média; $\mathrm{DP}=$ Desvio padrão.

de trabalho. Dentre as respostas que mais chamaram a atenção apontam-se: "... há um rigor grande com relação à higiene."; "Tudo é bem limpo..."; e "...tomo cuidado para que não aconteça (contaminação)".

Resultados semelhantes foram obtidos em trabalho realizado por Saccol et al. (2009) em 23 serviços de alimentação localizados no centro de Santa Maria/RS, onde foi aplicada uma lista de avaliação baseada na RDC 216/2004 por profissional técnico capacitado e pelo proprietário do estabelecimento ou responsável designado. Observou-se que, na avaliação realizada pelos proprietários, as empresas obtiveram um maior percentual de adequação das condições higiênicosanitárias quando comparadas com a avaliação realizada pelo profissional técnico capacitado. Isso demonstra que os proprietários possuem uma percepção distorcida de seu estabelecimento, o que para a autora indica que os responsáveis pelos serviços de alimentação estão acostumados com as não conformidades apresentadas ou não possuem capacitação técnica suficiente para estarem atuando no setor alimentício. Esses resultados demonstram, mais uma vez, a necessidade da capacitação de todos que trabalham nos serviços de alimentação a fim de assegurar a qualidade das refeições servidas.

Em relação à contaminação dos alimentos pelas mãos, embora os manipuladores saibam dessa possibilidade (94,2\% de respostas corretas), foi observado nos RPP estudados que a frequência da higienização das mãos é muito baixa e, além disso, verificou-se o uso de adornos (anéis) e esmalte nas unhas por esses profissionais.
Guimarães (2006), ao questionar os trabalhadores de restaurante comercial sobre a importância de praticar uma boa higienização das mãos, aponta que houve mais respostas em branco (21\%) no grupo dos trabalhadores não capacitados do que no grupo capacitado (1,5\%).

A importância da capacitação dos manipuladores sobre o procedimento correto de higienização das mãos pode ser observado em pesquisa realizada por Cruz et al. (2003) com manipuladores de quatro lanchonetes situadas ao redor do campus do CEFET/Química de Nilópolis/RJ e que não haviam recebido nenhum tipo de capacitação. Em avaliação por análise microbiológica, foi constatada uma precária qualidade higiênico-sanitária das mãos desses profissionais, sendo identificados coliformes totais e termotolerantes, alto índice de bactérias mesófilas e, ainda, Staphylococcos aureus. Esses resultados mostram que os manipuladores podem ser veículos de toxinfecções alimentares, o que pode comprometer a saúde dos consumidores. Logo, para que sejam servidas refeições em condições adequadas para consumo, faz-se necessário que os manipuladores apresentem boa higiene pessoal, principalmente no que se refere aos cuidados higiênicos das mãos.

Em relação ao conhecimento sobre DTA, 69\% dos 103 funcionários responderam incorretamente e, destes, $49,5 \%$ haviam sido capacitados. Os manipuladores confundem os tipos de DTA com sintomas gerais, como colocado a seguir: "Diarreia e qualquer outro tipo de doença."; "Dor no corpo, febre, vômito e diarreia." e "Dor no estômago, cabeça e diarreia." 
Resultado semelhante foi encontrado no trabalho realizado por Silva et al. (2003) com pessoas responsáveis pela merenda escolar de 24 unidades de ensino fundamental localizadas em São Paulo/SP, em que 62,5\% afirmaram não conhecer as DTA, sendo que, destes, $66,7 \%$ haviam sido capacitados.

Sugere-se que, durante a capacitação, sejam enfatizados os principais tipos de doenças causadas pelo consumo de alimentos contaminados e seus sintomas, para que os manipuladores adquiram o conhecimento adequadamente.

Foi verificado, ainda, o conhecimento dos manipuladores sobre o termo "Boas Práticas de Manipulação" e, dos 103 trabalhadores, 81,6\% disseram não saber o que significa, ou responderam incorretamente, mesmo tendo sido capacitados (66,7\%). Na maioria das vezes, o termo era confundido com a higiene pessoal como destacado a seguir: "... Roupa limpa, calçado"; "Pessoa que tem uma boa higiene no trabalho".

Segundo o nível de conhecimento dos manipuladores, baseado no parâmetro sugerido por Saccol (2007), nenhum dos RPP foi classificado como bom, $80 \%$ foram classificados como regulares e variaram de 51,6 a $67,9 \%$ as respostas corretas (Tabela1).

Dos 103 funcionários que responderam ao questionário, 69,9\% $(n=72)$ haviam sido capacitados (Tabela 1) e, destes, $53,4 \%(n=55)$ haviam recebido treinamento em um período de tempo igual ou menor que seis meses.

Os piores resultados em relação ao conhecimento sobre higiene pessoal, contaminação dos alimentos, doenças transmitidas por alimentos e boas práticas de manipulação foram nos RPP 3 e 10 que obtiveram, respectivamente, 51,7 e 50,8\% de respostas parcialmente corretas e incorretas (Tabela 1). No que se refere ao RPP 3 , este resultado pode estar atrelado ao fato do restaurante ter um baixo percentual de manipuladores treinados, 37,5\%. Já no RPP 10, a capacitação ministrada pode não estar sendo eficaz, pois, da amostra de manipuladores que responderam ao questionário, 78,6\% já haviam recebido algum tipo de treinamento sobre BPM (Tabela 1). Isso reforça a ideia da necessidade de se buscar formas mais eficazes de capacitação e de acompanhamento das atividades dos manipuladores de alimentos.

Quando correlacionado o nível de conhecimento dos manipuladores dos RPP com sua capacitação, foi observada correlação moderada positiva $(r=0,66)$ (Figura1).

Quando comparada a capacitação dos manipuladores dos RPP com as condições higiênicosanitárias destes restaurantes apontadas por Mello (2009), foi observada correlação fraca positiva $(r=0,52)$ (Figura 2), o que comprova que, apesar da importância

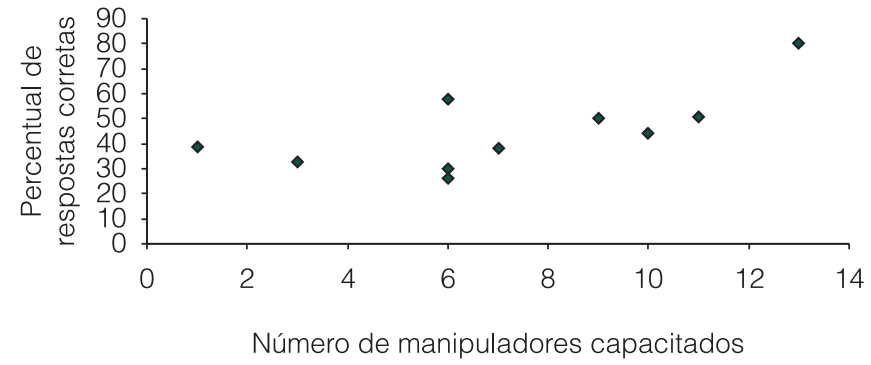

Figura 1. Nível de conhecimento dos manipuladores de alimentos sobre boas práticas de manipulação e existência de capacitação nos 10 RPP.

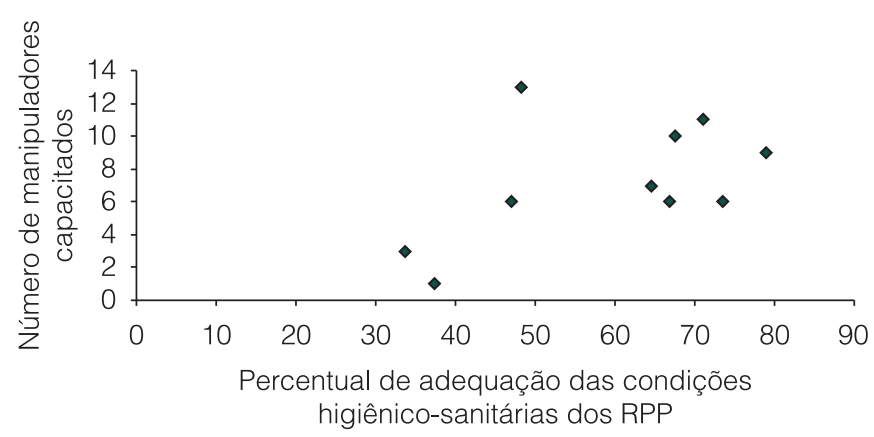

Figura 2. Capacitação dos manipuladores e percentual de adequação das condições higiênico-sanitárias dos 10 RPP.

da capacitação, outros fatores podem influenciar a inadequação das condições higiênico-sanitárias, como por exemplo, a estrutura física inapropriada, a falta de manutenção dos equipamentos e dos utensílios, como apontado por Mello (2009), e a alta rotatividade da mão de obra.

Esse resultado é reforçado por Panza et al. (2006), quando aponta um pequeno aumento no percentual de adequação das condições higiênico-sanitárias (de 23,7 para $36,5 \%$ ), após a capacitação dos trabalhadores, mostrando a interferência de outros fatores. No entanto, em pesquisa realizada por Guimarães (2006), os trabalhadores reconheceram que a capacitação facilita a execução das atividades nos serviços de alimentação com segurança.

\section{Conclusões}

Através dos resultados obtidos, pode-se verificar que os manipuladores de alimentos que trabalham nos RPP recebem baixos salários, sendo este um dos fatores que pode prejudicar a implantação das boas práticas de manipulação devido à grande rotatividade em busca de melhores salários. Observou-se também que estes manipuladores possuem baixo nível de escolaridade, sendo um desafio encontrar a melhor forma de capacitação desses profissionais a fim de atender às exigências da legislação vigente e fornecer refeições com segurança sob o aspecto higiênico-sanitário. 
Conhecimento dos manipuladores de alimentos sobre boas práticas nos restaurantes públicos populares do Estado do Rio de Janeiro

MELLO, A. G. et al.

No geral, o nível de conhecimento dos manipuladores sobre higiene pessoal, contaminação dos alimentos, doenças transmitidas por alimentos e boas práticas de manipulação é regular, o que pode contribuir para a contaminação das refeições produzidas e, consequentemente, o aparecimento de doenças transmitidas por alimentos. Em nenhum RPP o nível de conhecimento dos manipuladores foi considerado bom e os piores resultados foram obtidos pelos RPP 3 e 10.

Há uma correlação positiva entre as variáveis nível de conhecimento e capacitação dos trabalhadores dos RPP. Também pôde ser observada correlação positiva entre as variáveis capacitação dos trabalhadores dos RPP e o percentual de adequação das condições higiênicosanitárias.

Verificou-se, ainda, que o setor de alimentação coletiva contrata muitos profissionais sem experiência na função que irão desempenhar, logo, cabe investir na capacitação dos manipuladores de alimentos. É, contudo, um desafio encontrar a melhor didática para esta prática, de forma que não só atenda aos anseios dos trabalhadores, mas também às exigências da legislação sanitária.

Sugere-se que a supervisão seja reforçada durante o processo produtivo das refeições e que seja desenvolvido um programa de capacitação contínuo para os manipuladores de alimentos que trabalham nos RPP, a fim de que o conhecimento seja adquirido e aplicado no cotidiano e também para que o RPP alcance o seu objetivo final que é fornecer refeições com qualidade e segurança.

\section{Referências}

ALMEIDA, R. C. C.; KUAYE, A. Y.; SERRANO, A. M.; ALMEIDA, P. F. Avaliação e controle da qualidade microbiológica de mãos de manipuladores de alimentos. Revista de Saúde Pública, São Paulo, v. 29, n. 4, p. 290-94, 1995.

ARRUDA, G. A. Análise de perigos em pontos críticos de controle no SND. In: FERNANDES, A. T.; FERNANDES, M. O. V.; RIBEIRO FILHO, N. (Orgs.). Infecção hospitalar e suas interfaces na área de saúde. São Paulo: Atheneu, 2000. Disponível em: <http://www.ccih.med.br/novocapitulo66.html>. Acesso em: 12 de julho de 2006.

BARBOSA NETA, R. X.; HOLLAND, N.; DAMASCENO, K. S. F. S. C. Análise dos perigos e pontos críticos de controle durante o preparo da alface servida no restaurante universitário da UFRN. Revista Higiene Alimentar, São Paulo, v. 18, n. 126-127, p. 36-42, 2004.

BENEVIDES, C. M. J.; LOVATTI, R. C. C. Segurança alimentar em estabelecimentos processadores de alimentos. Revista Higiene Alimentar, São Paulo, v. 18, n. 125, p. 24-27, 2004
BRASIL. Agência Nacional de Vigilância Sanitária. Resolução RDC n. 216, de 15 de setembro de 2004. Estabelece procedimentos de boas práticas para serviços de alimentação a fim de garantir as condições higiênico-sanitárias do alimento preparado. Diário Oficial da União, Poder Executivo, Brasília, DF, 17 set. 2004.

CASTRO, F. T. Restaurantes do tipo self-service: análise dos aspectos sanitários e dos manipuladores de estabelecimentos localizados nos shoppings centers da cidade do Rio de Janeiro/ RJ. 2007. 105 f. Dissertação (Mestrado em Tecnologia dos Alimentos) - Universidade Federal Rural do Rio de Janeiro, Rio de Janeiro, 2007.

CRUZ, A. G.; LOUZA, B. J. G.; CORNO, C. N.; FERNANDEZFERREIRA, E.; TEIXEIRA, F. M.; SANTOS, G. O.; SOUZA, M. A. L.; MARTINS, O. R.; TAVARES, R. S.; TEIXEIRA, R. C. A questão da higiene de manipuladores das lanchonetes localizadas ao redor do campus do CEFET/Química de Nilópolis, RJ. Revista Instituto Adolfo Lutz, Rio de Janeiro, v. 62, n. 3, p. 245-248, 2003.

FERNÁNDEZ, M. E. L.; TORRES, A. C.; SELVA, M. C.; RODRÍGUEZ, F. S. Cómo educar en higiene de los alimentos. Revista Cubana de Alimentación y Nutrición, La Habana, v. 12, n. 1, p. 51-54, 1998. Disponível em: <http://bvs.sld.cu/ revistas/ali/vol12_1_98/ali10198.pdf>. Acesso em: 11 de junho de 2009.

GIL, A. C. Métodos e técnicas de pesquisa social. São Paulo: Atlas, 1998.

GUIMARÃES, K. A. S. Ações educativas para a promoção da saúde e da segurança do trabalho em restaurantes comerciais. 2006. 190 f. Dissertação (Mestrado em Biociências e Saúde) - Fundação Oswaldo Cruz, Rio de Janeiro, 2006.

LAGAGGIO, V. R. A.; FlORES, M. L.; SEGABINAZI, S. D. Avaliação microbiológica da superfície das mãos dos funcionários do restaurante universitário da Universidade Federal de Santa Maria, RS. Revista Higiene Alimentar, São Paulo, v. 16, n. 100, p. 107-110, 2002.

MELLO, A. G. Condições higiênico-sanitárias na produção de refeições em restaurantes públicos populares localizados no Estado do Rio de Janeiro. 2009. 130 f. Dissertação (Mestrado em Vigilância Sanitária). Fundação Oswaldo Cruz, Rio de Janeiro, 2009.

MINISTÉRIO DO DESENVOLVIMENTO SOCIAL E COMBATE À FOME - MDS. Pesquisa revela perfil de usuários de restaurantes populares. Brasília, 2005. Disponível em: <http://www.ibope.com.br/opiniao_publica/downloads/opp_ restaurantespopulares_set05.pdf $>$. Acesso em: 27 de maio de 2008.

ORGANIZAÇÃO MUNDIAL DE SAÚDE - OMS. Food safety and foodborne illness. Genebra, 2002. Disponível em: <http:// www.who.ont/mediacenter/factsheets/fs237>. Acesso em: 15 de junho de 2005. 
Conhecimento dos manipuladores de alimentos sobre boas práticas nos restaurantes públicos populares do Estado do Rio de Janeiro

MELLO, A. G. et al.

ORGANIZAÇÃO MUNDIAL DE SAÚDE - OMS. Métodos de vigilância sanitária y gestión para manipuladores de alimento. Informe de uma reunión de consulta de la OMS. Genebra, 1989. Disponível em: <http//:whqlibdoc.who.int/ trs/who_TRS_785_spa.pdf>. Acesso em: 18 de setembro de 2005.

PANZA, S. G. A.; SPONHOLZ, T. K. Manipulador de alimentos: um fator de risco na transmissão de enteroparasitoses? Revista Higiene Alimentar, São Paulo, v. 22, n. 158, p. 42-47, 2006.

PRAXEDES, P. C. G. Aspectos da qualidade higiênicosanitária de alimentos consumidos e comercializados na cidade de São Remo. 2003. 120 f. Dissertação (Mestrado em Epidemiologia Experimental e Aplicada ao Controle das Zoonoses) - Universidade de São Paulo, São Paulo, 2003.

SACCOL, A. L. F. Sistematização de ferramentas de apoio para boas práticas em serviços de alimentação. 2007. 188 f. Dissertação (Mestrado em Ciência e Tecnologia dos Alimentos) - Universidade Federal de Santa Maria, Rio Grande do Sul, 2007.

SACCOL, A. L. F.; STANGARLIN, L.; RICHARDS, N. S.; HECKTHEUER, L. H. Avaliação das boas práticas em duas visões: técnica e da empresa. Brazilian Journal of Food Technology, Campinas, n. 4, p. 19-23, 2009.

SECRETARIA DE ESTADO DE ASSISTÊNCIA SOCIAL E DIREITOS HUMANOS - SEASDH. Termo de referência: prestação de serviços de preparo, fornecimento e distribuição de alimentação a preços populares, no Restaurante Popular Central do Brasil. Rio de Janeiro, 2007

SECRETARIA DE ESTADO DE ASSISTÊNCIA SOCIAL E DIREITOS HUMANOS - SEASDH. Restaurante popular. Rio de Janeiro, 2008. Disponível em: <http://200.156.42.162/webpopular/>. Acesso em: 20 de novembro de 2008.

SILVA, C.; GERMANO, M. I. S.; GERMANO, P. M. L. Condições higiênico-sanitárias dos locais de preparação da merenda escolar, da rede estadual de ensino em São Paulo/SP. Revista Higiene Alimentar, São Paulo, v. 17, n. 110, p. 49-55, 2003.

SILVA JÚNIOR, E. A. Manual de controle higiênico-sanitário em alimentos. São Paulo: Ed. Varela, 2007. 623 p.

SOUZA, R. R.; GERMANO, P. M. L.; GERMANO, M. I. S. Técnica da simulação aplicada ao treinamento de manipuladores de alimentos, como recurso para a segurança alimentar de refeições transportadas. Revista Higiene Alimentar, São Paulo, v. 18, n. 122, p. 21-25, 2004.

VIEIRA, S. Metodologia científica para a área de saúde. São Paulo: Sarvier, 1984.

WEI, H. L.; CHIOUS, C. S. Molecular subtyping of Staphylococcus aureus from an outbreak associated with a food handler. Epidemiology and Infection, Cambridge, v. 128, n. 1, p. 15-20, 2002. 